\title{
Resonant Effects in Terahertz Generation with Laser-Induced Gas Plasmas
}

\author{
I. Thiele ${ }^{1,2}$, B. Zhou ${ }^{3}$, A. Nguyen ${ }^{4}$, E. Smetanina ${ }^{1,5}$, R. Nuter ${ }^{1}$, P. González de Alaiza \\ Martínez ${ }^{1}$, K. J. Kaltenecker ${ }^{3}$, J. Déchard ${ }^{4}$, L. Bergé ${ }^{4}$, P. U. Jepsen ${ }^{3}$, and S. Skupin ${ }^{1,6}$ \\ ${ }^{1}$ Univ. Bordeaux - CNRS - CEA, Centre Lasers Intenses et Applications, Talence, France \\ ${ }^{2}$ Department of Physics, Chalmers University of Technology, Göteborg, Sweden \\ ${ }^{3}$ DTU Fotonik, Technical University of Denmark, Kongens Lyngby, Denmark \\ ${ }^{4}$ CEA/DAM Ile-de-France, Bruyères-le-Châtel, 91297 Arpajon, France \\ ${ }^{5}$ Department of Physics, University of Gothenburg, Göteborg, Sweden \\ ${ }^{6}$ Institut Lumière Matière, Université Lyon 1 - CNRS, Villeurbanne, France, stefan.skupin@univ-lyon1.fr
}

\begin{abstract}
Research on intense terahertz $(\mathrm{THz})$ electromagnetic sources has received an increasing attention owing to numerous applications, for example, in timedomain spectroscopy, biomedical imaging or security screening [1]. Among the various techniques employed to generate $\mathrm{THz}$ radiation, focusing intense two-color femtosecond pulses in air or noble gases provides interesting features like absence of material damage, large generated bandwidth (up to $\sim 100 \mathrm{THz}$ ) and high amplitudes of the emitted $\mathrm{THz}$ pulses $(>100$ $\mathrm{MV} / \mathrm{m}$ ) [2]. First reported by Cook et al. [3], $\mathrm{THz}$ emission from intense two-color pulses was initially attributed to optical rectification via third-order nonlinearity. However, it was shown later that the plasma built-up by tunneling photoionization is necessary to explain the high amplitudes of the $\mathrm{THz}$ field [4], and a quasi-dc plasma current generated by the temporally asymmetric two-color field is responsible for $\mathrm{THz}$ emission [5].
\end{abstract}

Numerous experimental results show that the laser-induced free electron density has a strong impact on the $\mathrm{THz}$ emission $[4,6,7]$. While it is frequently observed that a larger free electron density leads to broader $\mathrm{THz}$ spectra, the origin of the effect remains controversial. In $[6,7]$, homogeneous plasma oscillations were proposed as an explanation, even though those oscillations are in principle non-radiative $[8,9]$. Moreover, nonlinear propagation effects have been held responsible for $\mathrm{THz}$ spectral broadening as well [10]

On the other hand, the gas plasma produced by the fs laser pulse is a finite conducting structure with a lifetime largely exceeding the fs time scale. Thus, one can expect that the gas plasma features plasmonic resonances which may have a strong impact on the $\mathrm{THz}$ emission propertie [11]. However, no direct evidence of plasmonic effects in laser-induced gas-plasmas was observed so far: To make an evidence of plasmonic effects, those need to be distinguished from nonlinear propagation effects. Also from the theoretical point of view capturing plasmonic effects is not trivial: plasmonic effects require at least a full two-dimensional Maxwell-consistent description, and reduced models like the unidirectional pulse propagation equation [12], which are frequently used to describe plasma-based $\mathrm{THz}$ generation $[5,7,10]$, are by construction not capable of capturing such resonant effects.

In this work, we consider the two-color-laser-induced plasma as a plasmonic structure, and investi- gate under which conditions such plasmonic perspective is important. In the context of plasmonic nanoantennas (or metamaterials), e.g, for second-harmonic generation, tailoring plasmonic resonances by tuning the shape of the plasmonic particle is a standard approach. Therefore, we follow a similar strategy and modify the usually prolate spheroidal plasma shape. Going to tri-axial ellipsiods which can be achieved by using elliptically shaped laser beams turns out to be already sufficient [13]. Depending on whether the laser polarization is oriented along the long beam axis (quasi transverse electric, qTE) or along the short beam axis (quasi transverse magnetic, qTM), plasmonic resonances are triggered or not (see Fig. 1).
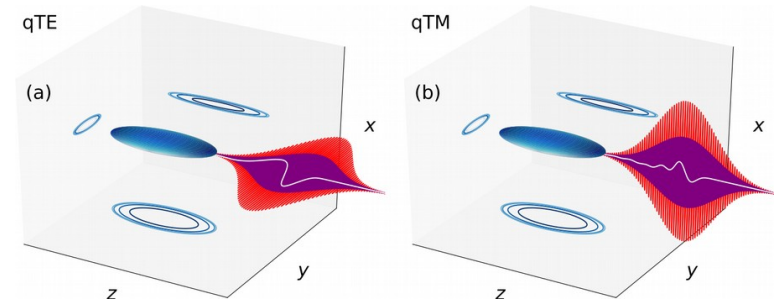

Fig. 1. Illustrated configurations of ${ }^{z} \mathrm{THz}$ emission from an ellipsoidal plasma induced by a two-color Gaussian laser pulse $\sim$ (funamental in red, second-harmonic in purple) with strongly elliptical beam shape propagating along $z$. The laser electric field is y-polarized (along the long axis of the beam, qTE) in (a) and x-polarized (along the short axis, qTM) in (b). The plasma is sketched as blue surface. Simulated forward emitted $\mathrm{THz}$ pulses are presented as white lines demonstrating a significantly shorter pulse duration for qTM polarization, which can be attributed to triggering a plasmonic resonance.

While nonlinear propagation effects are in both cases equally present, any difference between the $\mathrm{THz}$ emission spectra in this two cases is linked to plasmonic effects. We demonstrate experimental results which reveal a significant difference: $\mathrm{THz}$ pulses are shorter and have a broader emission spectrum when the plasma is excited by the laser field in the direction with the short focal beam width and plasma width (see Fig. 2). A simple analytical model allows us to link the broadening to a leaky mode. It turns out that the resonance has a strong impact on the spectrum whenever electrons are excited along a direction where the plasma size is smaller than the plasma wavelength. Finally, direct three-dimensional (3D) Maxwell consistent simulations in tightly focused geometry confirm that these plasmonic resonances indeed broaden the emitted $\mathrm{THz}$ spectrum significantly. 


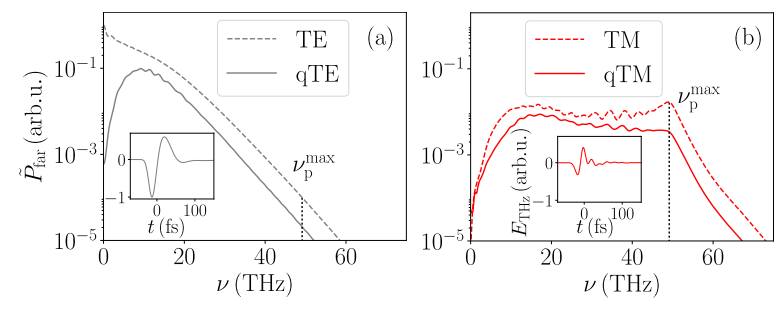

Fig. 2. Experimental THz spectra for qTE (a) and qTM (b) polarization (see text for details). Corresponding on-axis THz waveforms are shown as insets. The dashed lines specify the estimated maximum plasma frequency.

\section{References}

[1] M. Tonouchi. Cutting-edge terahertz technology. Nature Photon., 1:97, 2007.

[2] K. Kim et al. High-Power Broad-band Terahertz Generation via Two-Color Photo-ionization in Gases. IEEE J. Quant. Electron., 48:797, 2012.

[3] D. Cook et al. Intense terahertz pulses by four-wave rectification in air. Opt. Lett., 25:1210, 2000.

[4] K. Kim et al. Coherent control of terahertz supercontinuum generation in ultrafast laser-gas interactions. Nature Photon., 2:605, 2008.
[5] L. Bergé et al. 3D numerical simulations of $\mathrm{THz}$ generation by two-color laser filaments. Phys. Rev. Lett., 110:073901, 2013.

[6] H. Hamster et al. "Short-pulse terahertz radiation from high-intensity-laser-produced plasmas," Phys. Rev. E, 49:671, 1994

[7] V. A. Andreeva et al. Ultrabroad terahertz spectrum generation from an air-based filament plasma," Phys. Rev. Lett., 116:063902, 2016.

[8] V. T. Tikhonchuk, Comment on "generation of electromag-netic pulses from plasma channels induced by femtosecond light strings", Phys. Rev. Lett., 89:209301, 2002.

[9] I. Thiele et al. Theory of terahertz emission from femtosecond-laser-induced microplasmas, Phys. Rev. E, 94:063202, 2016.

[10] E. Cabrera-Granado et al. "Spectral self-action of thz emission from ionizing two-color laser pulses in gases, New J. Phys., 17:023060, 2015.

[11] V. A. Kostin at al. De to ac field conversion due to leaky-wave excitation in a plasma slab behind an ionization front, New J. Phys., 17:033029, 2015.

[12] M. Kolesik at al., Nonlinear optical pulse propagation simulation: From Maxwell's to unidirectional equations, Phys. Rev. E, 70:036604, 2004.

[13] I. Thiele et al. Terahertz emission from laserdriven gas-plasmas: a plasmonic point of view. arXiv:1803.06889 [physics.optics], 2018. 